$\mathrm{n}: 1)$, anti-Ro carriers (n:2 and n:1). No difference in complications between anti-Ro positive and negative women ( $p$ $=0.047$ ).

Hydroxicloroquine prescribed in 97 patients (64\%), aspirin in $99(65,6 \%)$, heparin in $33(21,9 \%)$ and prednisone in 48 (31\%).

Conclusions In our series, women with SLE and APL have higher risk of abortion, pregnancy complications and instrumental delivery than general population. Anti-Ro carriers don't have increased rate of abortions nor complications during pregnancy. Follow-up of pregnancy in a multidisciplinary unit decreases the risk of abortion.

\section{P82 ANTI-PHOSPHOLIPID ANTIBODIES AND RENAL INVOLVEMENT ARE THE MAIN FEATURES ASSOCIATED WITH ADVERSE PREGNANCY OUTCOMES IN PATIENTS WITH SYSTEMIC LUPUS ERYTHEMATOSUS - A LONG- TERM LONGITUDINAL STUDY IN SOUTHERN SWEDEN}

\footnotetext{
${ }^{1}$ Muna Saleh, ${ }^{2}$ Andreas Jönsen, ${ }^{2}$ Anders Bengtsson, ${ }^{1}$ Christopher Sjöwall, ${ }^{2}$ Michele Compagno. 'Dept. of Rheumatology, University hospital, Linköping; ${ }^{2}$ Dept. of Clinical Sciences, Rheumatology, Skåne university Hospital, Lund, Sweden
}

\subsection{6/lupus-2020-eurolupus. 127}

Background Systemic Lupus Erythematosus (SLE) affects mostly women in childbearing age. Modern management of SLE patients has improved the pregnancy outcomes over the last decades. However, there is still an increased risk of maternal, fetal and neonatal complications. In this longitudinal follow-up of pregnant women affected by SLE, we aimed to investigate which clinical and immunological features may predict for the occurrence of adverse pregnancy outcomes (APOs).

Methods We investigated the outcome of 59 pregnancies in 28 SLE patients who have had one or more pregnancies, between 2002 and 2018. Longitudinal clinical and laboratory data from rheumatology, obstetrics and neonatal units were collected and analyzed. We assessed the association between the presence of SLE-related clinical and immunological features and the occurrence of adverse pregnancy outcomes.

Results We recorded 52 APOs in $18(64.3 \%)$ patients. The 59 investigated gestations resulted in 44 (31 vaginal and $13 \mathrm{C}$ sections) deliveries, $8(18.2 \%)$ before the 37 th gestational week, $13(22 \%)$ early miscarriages and $2(3.4 \%)$ induced abortions. HELLP syndrome and preeclampsia complicated 1 $(2.3 \%)$ and $11(25 \%)$ gestations, respectively. Moreover, 10 (22.7\%) newborns had low birth weight, 5 (11.4\%) fetuses had intra-uterine growth restriction, whereof 1 (2.3\%) resulted in small for gestational age neonate. Neonatal lupus occurred in $1(2.3 \%)$ baby. Previous lupus nephritis was associated with higher risk of APOs overall $(\mathrm{OR}=5.9-p=0.01)$, in particular impaired fetal growth $(\mathrm{OR}=16.6-p=0.01)$. The presence of anti-phospholipid antibodies was also associated with higher risk of APOs overall $(\mathrm{OR}=4.5-p=0.01)$. In particular, the occurrence of preterm delivery and the incidence of miscarriage were associated with the presence during pregnancy of anti-cardiolipin antibodies $(\mathrm{OR}=6.8-p=0.03)$ and with concomitant anti-phospholipid syndrome (APS) $(\mathrm{OR}=3.3-p=0.04)$, respectively.

Conclusions Several different APOs occur in the majority of SLE-patients, in particular in those with renal involvement, APS and presence of anti-phospholipid antibodies.

\section{P83 \\ A PRE-PREGNANCY COUNSELLING PATHWAY FOR WOMEN WITH SYSTEMIC LUPUS ERYTHEMATOSUS AND/OR ANTIPHOSPHOLIPID SYNDROME: THE EFFECT ON MATERNAL AND FETAL PREGNANCY COMPLICATIONS AND THE COURSE OF DISEASE - A RETROSPECTIVE COHORT STUDY}

${ }^{1}$ Merlijn Wind, ${ }^{1}$ Maike Hendriks, ${ }^{1}$ Marieke Sueters, ${ }^{2}$ Onno Teng. 'Dept. of Obstetrics, Leiden University Medical Center (LUMC), Leiden; ${ }^{2}$ Dept. of Nephrology, LUMC, Leiden, The Netherlands

\subsection{6/lupus-2020-eurolupus. 128}

Background Women with systemic lupus erythematosus (SLE) and/or antiphospholipid syndrome (APS) are at higher risk of complications and SLE flares during pregnancy and are therefore referred to as 'high risk pregnancies'. To provide optimal patient care, a multidisciplinary pre-pregnancy counselling approach is recommended. We examined the effect of such a multidisciplinary pre-pregnancy counselling pathway that is specifically designed for SLE and/or APS patients on the maternal and fetal pregnancy complications and on the course of SLE and APS disease.

Methods We performed a retrospective cohort study on records in the Leiden University Medical Center (LUMC), a tertiary referral hospital in the Netherlands. We compared a cohort of SLE and/or APS pregnancies enrolled in the prepregnancy counselling pathway (2014-2018) with a historical cohort of SLE and/or APS pregnancies that were not enrolled in the pathway (2008-2014).

Results This study was done on 34 pregnancies in the pathway cohort and 71 in the cohort. The pathway cohort had more severe SLE disease than the historical cohort. SLE flares developed in $18(32 \%)$ of all SLE pregnancies, whereas the risk on a flare was significantly $(p=0.042)$ lower in the pathway cohort $(n=1,8 \%)$ than in historical cohort $(n=17,40 \%)$. The incidence of maternal and fetal pregnancy complications were not different between the pathway and the historical cohort.

Conclusions This study showed the positive effect of a multidisciplinary pathway for SLE and/or APS patients on a significant reduction in SLE flares. Although, overall, the patients that were enrolled in the pathway suffered from more severe SLE disease than the historical cohort, the incidence of maternal and fetal complications were similar in both groups. We believe that, women with SLE and/or APS would benefit from pre-pregnancy referral to a hospital with a multidisciplinary approach towards pre-pregnancy counselling and pregnancy follow up.

\section{P84 LUPUS AND PREGNANCY IN URUGUAY: SUCCESSFUL OUTCOMES IN AN INTEGRATED CARE UNIT}

Valentina Zubiaurre, Carolina DeLos Santos, Mariana Cora, Valentin Gonzalez Sebastián Rosano, Ricardo Silvariño, Leonardo Sosa. Departamento de Medicina, Hospital de Clínicas, UDELAR, Montevideo, Uruguay

\subsection{6/lupus-2020-eurolupus.129}

Background/Purpose Most reports show an increased Systemic Lupus Erythematosus (SLE) activity during pregnancy and worse maternal-fetal outcomes than those of the general population. The objective of this work is to describe pregnancy outcomes of Uruguayan women with SLE. 